\title{
Efficient gene editing through an intronic selection marker in cells
}

\author{
Shang Wang ${ }^{1,2} \cdot$ Yuqing $\mathrm{Li}^{2,3} \cdot \mathrm{Li}^{\mathrm{Zhong}}{ }^{4,5} \cdot \mathrm{Kai}^{\mathrm{W}} \mathrm{u}^{2} \cdot$ Ruhua Zhang ${ }^{1} \cdot$ Tiebang Kang ${ }^{1} \cdot$ Song $\mathrm{Wu}^{2,3,6} \cdot$ Yuanzhong Wu${ }^{1} \mathbb{C}$
}

Received: 21 July 2021 / Revised: 10 January 2022 / Accepted: 14 January 2022 / Published online: 31 January 2022

(C) The Author(s) 2022

\begin{abstract}
Background Gene editing technology has provided researchers with the ability to modify genome sequences in almost all eukaryotes. Gene-edited cell lines are being used with increasing frequency in both bench research and targeted therapy. However, despite the great importance and universality of gene editing, the efficiency of homology-directed DNA repair (HDR) is too low, and base editors (BEs) cannot accomplish desired indel editing tasks.

Results and discussion Our group has improved HDR gene editing technology to indicate DNA variation with an independent selection marker using an HDR strategy, which we named Gene Editing through an Intronic Selection marker (GEIS). GEIS uses a simple process to avoid nonhomologous end joining (NHEJ)-mediated false-positive effects and achieves a DsRed positive rate as high as $87.5 \%$ after two rounds of fluorescence-activated cell sorter (FACS) selection without disturbing endogenous gene splicing and expression. We re-examined the correlation of the conversion tract and efficiency, and our data suggest that GEIS has the potential to edit approximately $97 \%$ of gene editing targets in human and mouse cells. The results of further comprehensive analysis suggest that the strategy may be useful for introducing multiple DNA variations in cells.
\end{abstract}

Keywords Gene editing $\cdot$ HDR $\cdot$ Intron reporter $\cdot$ FACS $\cdot$ Conversion tract

\section{Background}

Shang Wang, Yuqing Li and Li Zhong are joint first authors.

Song Wu

wusong@mail.szu.edu.cn

$\checkmark$ Yuanzhong Wu

wuyzh@sysucc.org.cn

1 State Key Laboratory of Oncology in South China, Collaborative Innovation Center for Cancer Medicine, Department of Experimental Research, Sun Yat-Sen University Cancer Center, Guangzhou 510060, China

2 Institute of Urology, The Third Affiliated Hospital of Shenzhen University, Shenzhen 518000, China

3 Teaching Center of Shenzhen Luohu Hospital, Shantou University Medical College, Shantou 515000, China

4 Center of Digestive Diseases, The Seventh Affiliated Hospital of Sun Yat-Sen University, Shenzhen 518107, China

5 Scientific Research Center, The Seventh Affiliated Hospital of Sun Yat-Sen University, Shenzhen 518107, China

6 Department of Urology, South China Hospital of Shenzhen University, Shenzhen 518000, China
Genetic mutations cause human diseases, including cancers and heritable disorders. Therefore, gene editing technologies that can easily correct or generate mutations are critical for clinical applications and bench research, respectively [1]. Prokaryotes-derived CRISPR-Cas systems mediate target DNA cleavage guided by protospacer adjacent motif (PAM) and sgRNAs that form the DNA-RNA heteroduplex with the target genome DNA. Cas 12 and Cas9 have been widely applied for eukaryotic genome editing because of their high efficiency in generating DNA double-strand breaks (DSBs), after which the DNA is repaired. Despite the potential for precise base editing by DNA repair, such as homologydirected repair (HDR) and nonhomologous end joining (NHEJ) [2], the efficiency of these methods varies dramatically in different cell lines [3, 4].

Recently, an alternative gene editing strategy was developed that uses dead Cas9 or Cas9 nickase to target DNA via sgRNA and recruits base deaminase domains to accomplish C-to-T base conversions (with a cytosine base editor, CBE) or A-to-G base conversions (with an adenine base editor, ABE) without introducing DSBs. Although the efficiency of this strategy can reach as high as $60 \%$, its off-target effects, 
inability to accomplish A-to-C, A-to-T, G-to-C or G-to-T conversion [5], and bystander effect might inhibit its application $[6,7]$.

Introns are transcribed together with exons as pre-mRNA but are spliced by the spliceosome complex so that mature mRNA does not contain intronic sequences [8]. Introns are frequently used as targets for HDR genome editing strategies $[9,10]$. To assist in the retrieval of successfully repaired clones, the target gene coding sequence (CDS) can be tagged with a fluorescent protein at the $\mathrm{N}$ - or $\mathrm{C}$-terminus as a selectable marker [11]. To remove these tags, seamless repair using piggyBac or sleeping beauty is available [12, 13]. However, this kind of selection can be used only when the target sites are near the terminus, and it changes the open reading frame (ORF), which may have unpredictable negative effects on genetic regulation. Furthermore, the marker is driven by the endogenous promoter, which might be too weak to make a difference for selection.

To solve the shortcomings of the current gene editing methods, we established an efficient gene editing system based on HDR-mediated intronic fluorescent protein insertion without disruption of endogenous gene splicing and expression, which we named Gene Editing through an Intronic Selection marker (GEIS). This strategy avoids donor DNA-mediated false positive cell clones and produces as many as $87.5 \%$ gene-edited cells in our tested loci. The results of further studies reveal its strong potential for use in 97\% of exon editing applications and for multiple mutation introduction.

\section{Results}

\section{The GEIS workflow generates RELA/p65 S276C HEK293T cells within 1 month}

p65 is a REL-associated protein involved in NF- $\mathrm{KB}$ heterodimer formation, nuclear translocation, and downstream gene transactivation [14]. We applied GEIS to generate the S276C mutation in RELA/p65. A LentiCRISPR-v2 plasmid carrying sgRNA targeting intron 8 of the RELA gene was used to generate DSBs. To avoid disrupting RNA splicing, we did not target the splice site. The donor DNA template contained a cytomegalovirus (CMV) promoter-driven DsRedexpressing cassette between the left and right homology arms (HAs), while the desired S276C mutation was located on the left arm (Fig. 1A). The LentiCRISPR-v2 plasmid and donor DNA were cotransfected into HEK293T cells for $24 \mathrm{~h}$, and then puromycin selection was conducted for $72 \mathrm{~h}$ to kill nontransfected cells. The surviving cells were subjected to FACS to fractionate the DsRed-positive cells. To increase the selection efficiency, a second round of FACS was performed. The sorted cells were seeded into 96 -well plates for single-cell clone growth. We obtained positive cell clones with the S276C mutation within 1 month with this workflow (Fig. 1B, C). Reverse transcription PCR (RT-PCR) and quantitative PCR (qPCR) showed that the inclusion of the CMV-DsRed cassette in the intron neither disturbed the splicing of the two adjacent exons nor affected mRNA transcription (Fig. 1D, E).

\section{HDR with an ssDNA template reduces the production of false-positive cell clones}

In this strategy, the use of dsDNA as donor DNA produces false-positive cell clones via direct transcription and translation or via random integration into the genome through canonical NHEJ (c-NHEJ) [15]. Recent studies have demonstrated that ssDNA donors show superior performance compared to dsDNA donors in mammalian systems by reducing the probability of NHEJ [16]. To effectively obtain ssDNA sequences as large as $5000 \mathrm{nt}$, we denatured the dsDNA from PCR at $95{ }^{\circ} \mathrm{C}$ and with $100 \mathrm{mM} \mathrm{NaCl}$ for 10 min (Fig. 2A). Transfection of dsDNA or ssDNA without CRISPR-Cas9 demonstrated that a single-stranded CMVDsRed donor led to significantly lower fluorescence intensity than a double-stranded donor. Considering that $\mathrm{NaCl}$ might influence transfection, we purified DNA. The purification of ssDNA resulted in a slight increase in DsRed-positive cells (Figs. 2B, S1A, B). Based on the above results, we speculated that the use of an ssDNA donor would increase the true-positive rate of FACS-enriched DsRed-expressing cells. As shown in Fig. 2C, with ssDNA as a donor cotransfected with CRISPR-Cas9, the recombination rate for RELA reached $87.5 \%$ (21 out of 24 ), while with dsDNA, it was only $41.7 \%$ (10 out of 24 ) after two rounds of sorting. Elevated recombination rates were also observed at the $N A B P 2$ and EGFR loci, and no abnormal splicing or mRNA level changes were detected (Fig. 2C-E).

\section{Conversion tract length influence gene editing efficiency}

Despite the efficient selection of successfully recombined cells, GEIS still exhibits a low efficiency when the conversion tract is too long. Because the CMV-DsRed cassette must be located in an intron to avoid disrupting endogenous gene splicing and expression, the sgRNA target site should usually be intronic, but the expected conversion site is usually exonic. The distance from the DSB to the conversion site (conversion tract) affects the efficiency [17].

To estimate the influence of conversion tract length on efficiency, we first evaluated the HA length required for efficient insertion of the selection cassette into the intron. Using the EGFR locus as an example, we designed a series of donors with 250, 500, 800 and 1000 nt HAs. We found that 
A

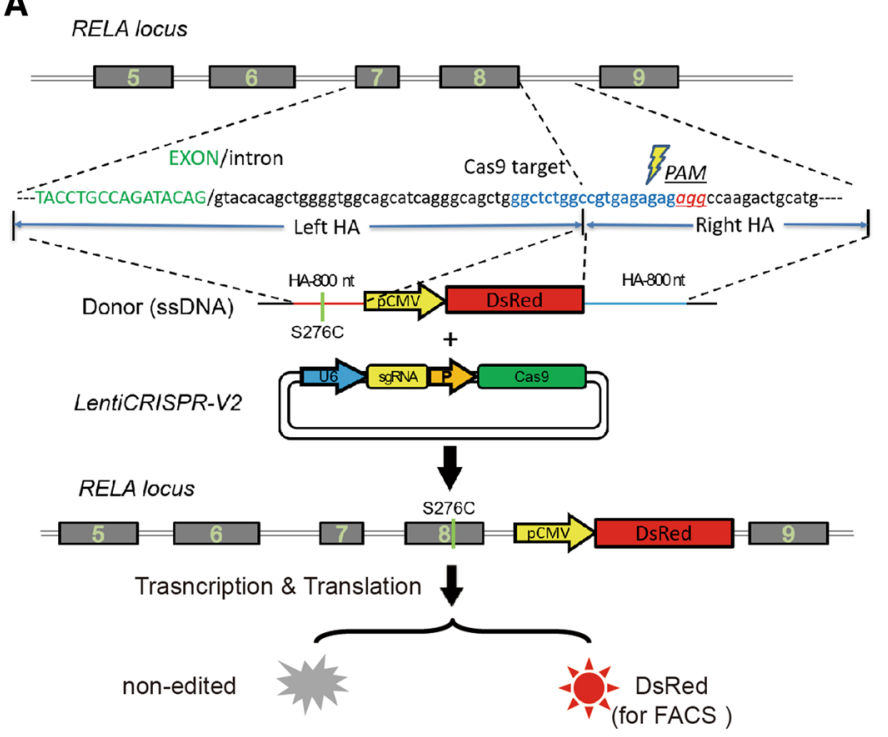

B

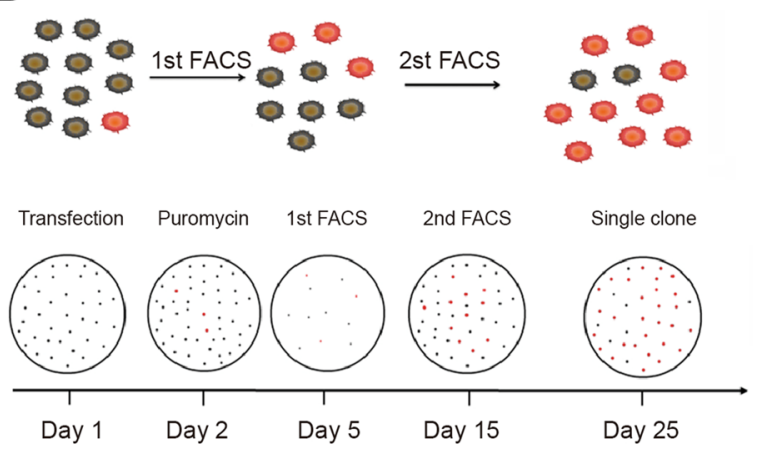

Fig. 1 A Schematic view of GEIS to introduce the S276C mutation into the RELA locus in HEK293T cells. LentiCRISPR-V2 is used to generate DSBs in intron 8 and donor ssDNA as a template to introduce mutations by HDR. B Workflow of GEIS, with two rounds of FACS to enrich and fractionate individual DsRed-positive cells. C Sanger sequencing of RELA genomic DNA sequences derived from

HAs longer than $500 \mathrm{nt}$ were necessary for recombination at this locus (Fig. S2 A, B). Next, we designed donor DNA with a left HA ( $800 \mathrm{nt})$ containing nucleotide variations 45 , 90, 171, 386, 490, 596 and 696 nt away from the DSB site for GEIS of NABP2 (Fig. 3A). The genomic DNA of the GEIS-processed cell group was PCR-amplified with the forward primer located outside the left HA on the genome and the reverse primer at the DsRed cassette. The PCR product was cloned into pLV-MCS-puro-Green for Sanger sequencing. A total of 624 amplicons were sequenced, and the conversion efficiency was calculated. We repeated this workflow in RELA and EGFR recombination to test more loci, which introduce nucleotide switches, insertions and deletions
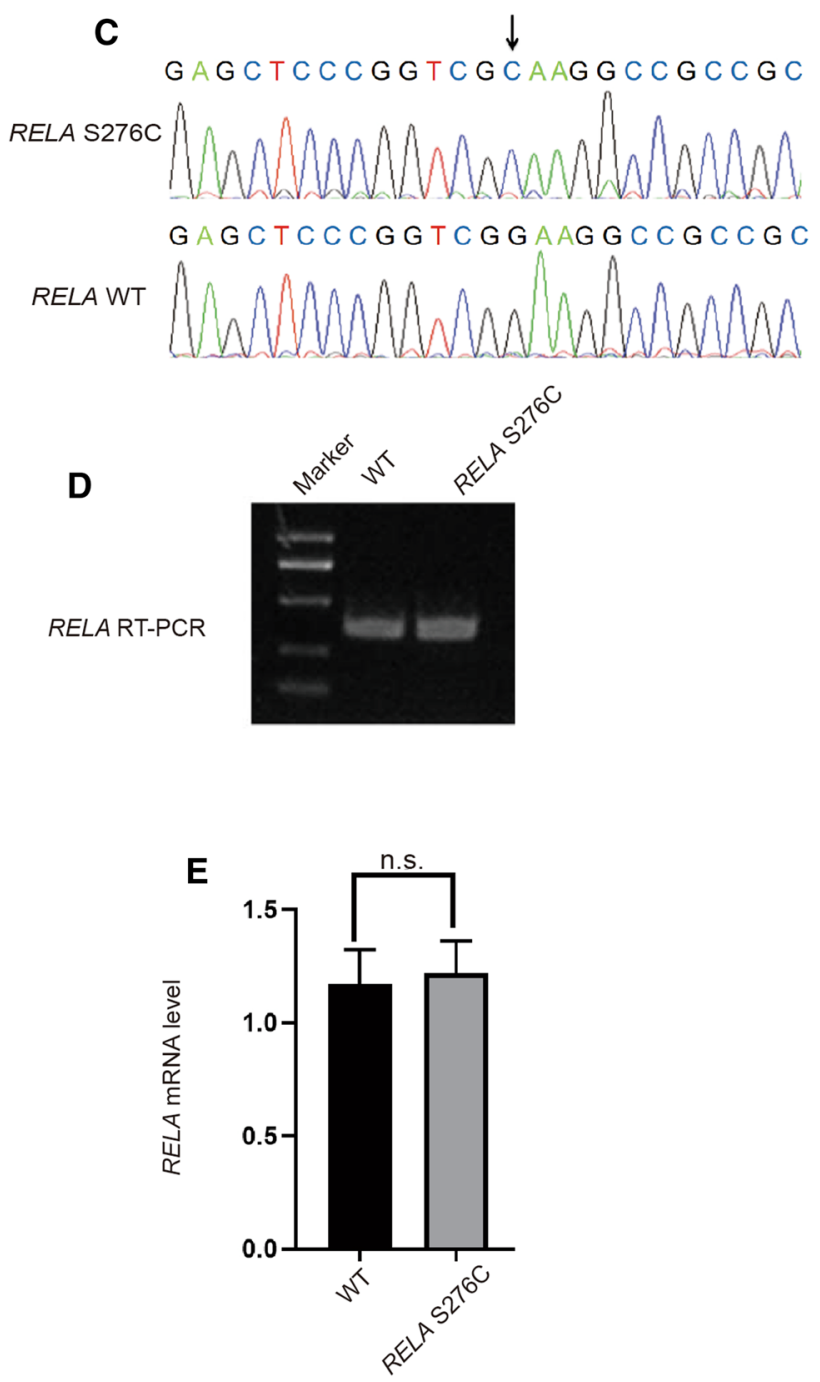

WT- and GEIS-treated RELA S276C gene-edited HEK293T cells. D RT-PCR of cDNA from WT and S276C HEK293T cells. No alternative variants were found. E Relative expression of RELA in WT and S276C HEK293T cells. No significant (n. s.) change in RELA expression was detected

(sequences are provided in the supplementary material). A total of 265 amplicons of EGFR and 446 amplicons of RELA were sequenced and calculated. In general, the conversion efficiency decreased as the tract became further away from the DSB. Although the efficiency varied largely in different loci, the conversion efficiency remained at $35 \%$ when the tract was shorter than $300 \mathrm{bp}$ in the tested loci (Fig. 3B). We picked single-cell clones from RELA- and EGFR-edited cells for Sanger sequencing to exclude the effect of false amplicons generated by the possible overlap-extension process in PCR amplification. The results were highly consistent with those of amplicons (Fig. S2C). Previous research based on 80 cell clones reported an efficiency of only $20 \%$ when the 


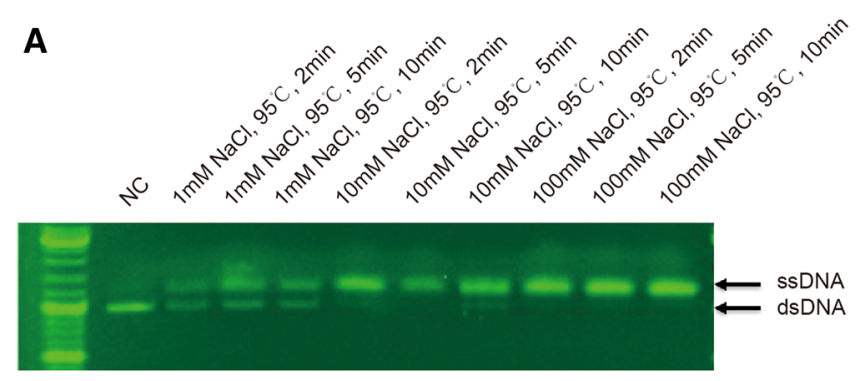

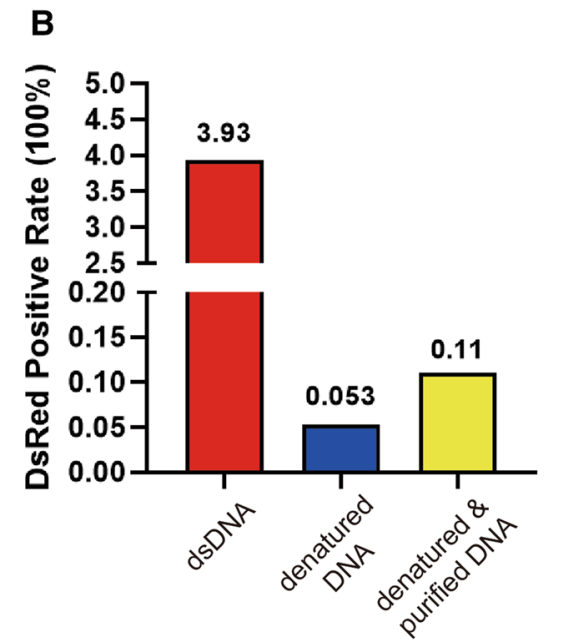
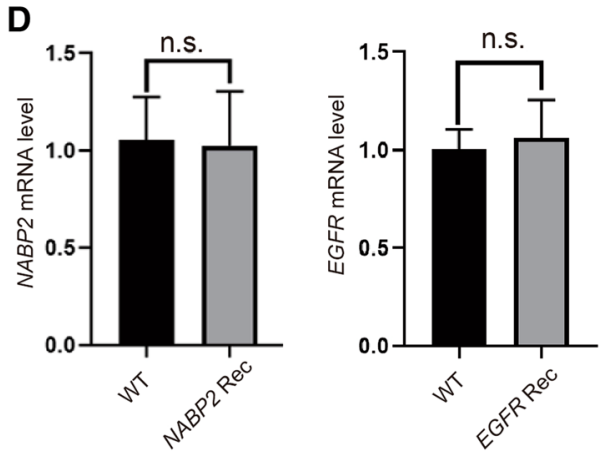

Fig. 2 A Agarose gel electrophoresis of denaturing dsDNA under the indicated conditions. B Percentage of DsRed-positive cells from $1 \mu \mathrm{g}$ of dsDNA-, ssDNA(denatured)- or ssDNA (denatured and purified)transfected HEK293T cells determined by FACS. C HDR efficiency of RELA, NABP2 and EGFR using GEIS using dsDNA (nondenatured) or ssDNA (denatured) as donor DNA. D. qPCR of $N A B P 2$

tract was $200 \mathrm{bp}$ long [17]. The extremely low efficiency might result from the DNA locus. Despite the variations, the three loci in this study showed far higher efficiencies than previously reported.

Because introns adjacent to the target exon on both the left and right sides are available for GEIS DSB generation, a nearer intron can always be found for the exonic editing site for GEIS, which needs less than half of the exon length as the conversion tract. For example, the conversion tract of $300 \mathrm{bp}$ indicated that GEIS has at least a $35 \%$ probability

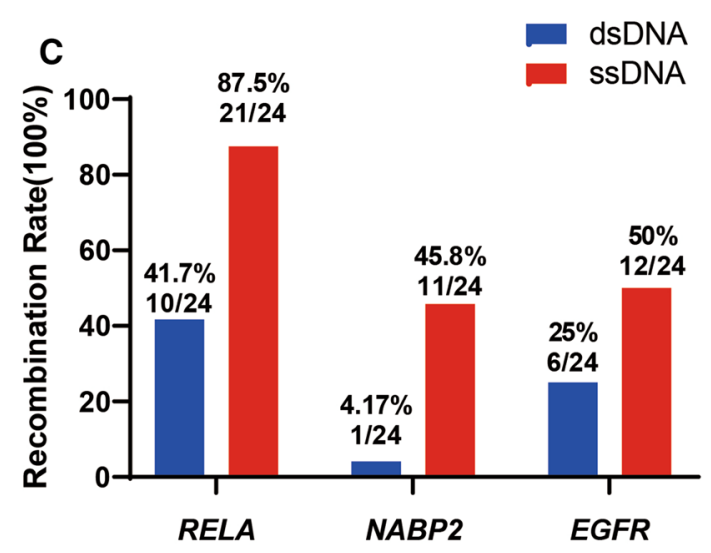

E

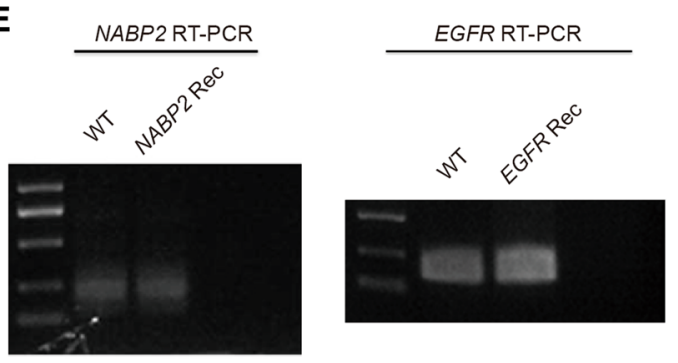

and EGFR in WT and GEIS-recombined (Rec) cells. Data are the mean \pm s.d. of $n=3$ biological independent experiments. No significant (n. s.) variation was found by Student's t-test for either NABP2 or $E G F R$ mutants. E Agarose gel electrophoresis of RT-PCR products of NABP2 and EGFR in WT- and GEIS-modified cells. No alternative variant was found of generating mutations for exons as large as $600 \mathrm{bp}$ in the locus (Fig. S2D).

Furthermore, we investigated the requirement of conversion lengths in gene editing tasks. To assess the applicability of GEIS in the human and mouse genomes, we analyzed the distributions of exon length in these two species from the Consensus CDS (CCDS) Project (Fig. S2E) [18-20]. Most exons longer than $600 \mathrm{bp}$ were the first or last exons, which contain long $5^{\prime}$ or $3^{\prime}$ untranslated regions (UTRs); however, DSBs can still be introduced by sgRNA in the first 
A

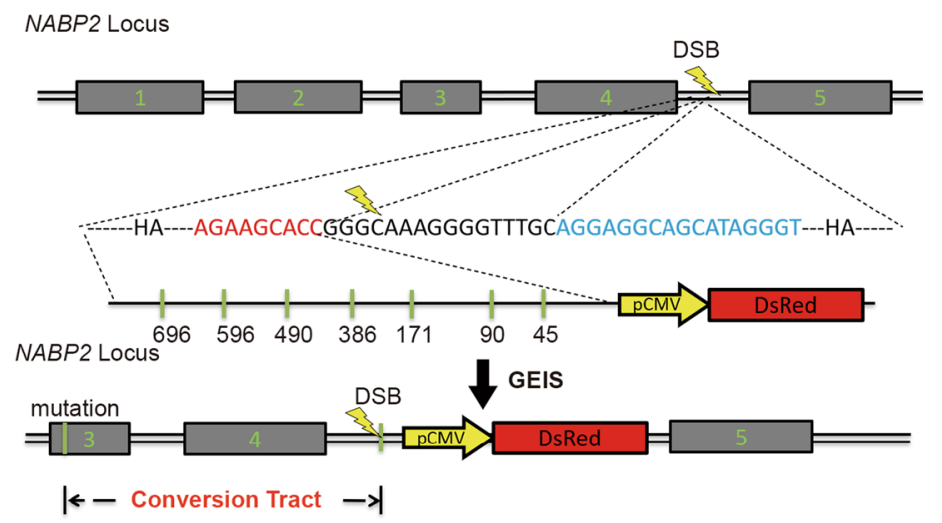

c

C Exon Length Distribution (UTR Excluded)

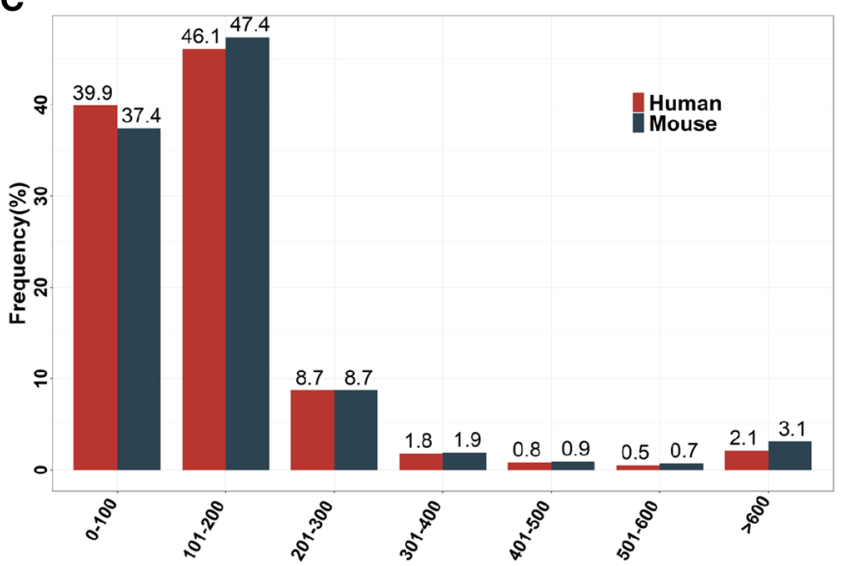

Fig. 3 A Schematic overview of the genome editing conversion tract experiments in the $N A B P 2$ locus: seven different DNA variations were included in the left HA of donor DNA in GEIS to test how many of these variations could be introduced in the genome locus of $N A B P 2$. B Percentage of DNA variations from the workflow of $\mathbf{A}$ in $N A B P 2, R E L A$ and EGFR that were introduced in the genome based on PCR amplicons. C Calculation of exon lengths of all human and

or last intron. When we excluded the UTRs and reanalyzed the distribution of exon lengths, only approximately $3 \%$ of exons had lengths longer than $600 \mathrm{bp}$ (Fig. 3C). Based on the conversion tract analysis from the NABP2, RELA and EGFR loci, we speculate that GEIS might be able to edit $97 \%$ of gene targets with relatively high efficiency.

\section{GEIS has the potential to introduce multiple DNA variations}

To evaluate the possibility of introducing multiple genome alterations in one GEIS reaction, we analyzed the mutation distributions in each of the 624 NABP2 amplicons. A heatmap was created to show the percentage of alterations that occurred at the remaining sites (horizontal axis) when
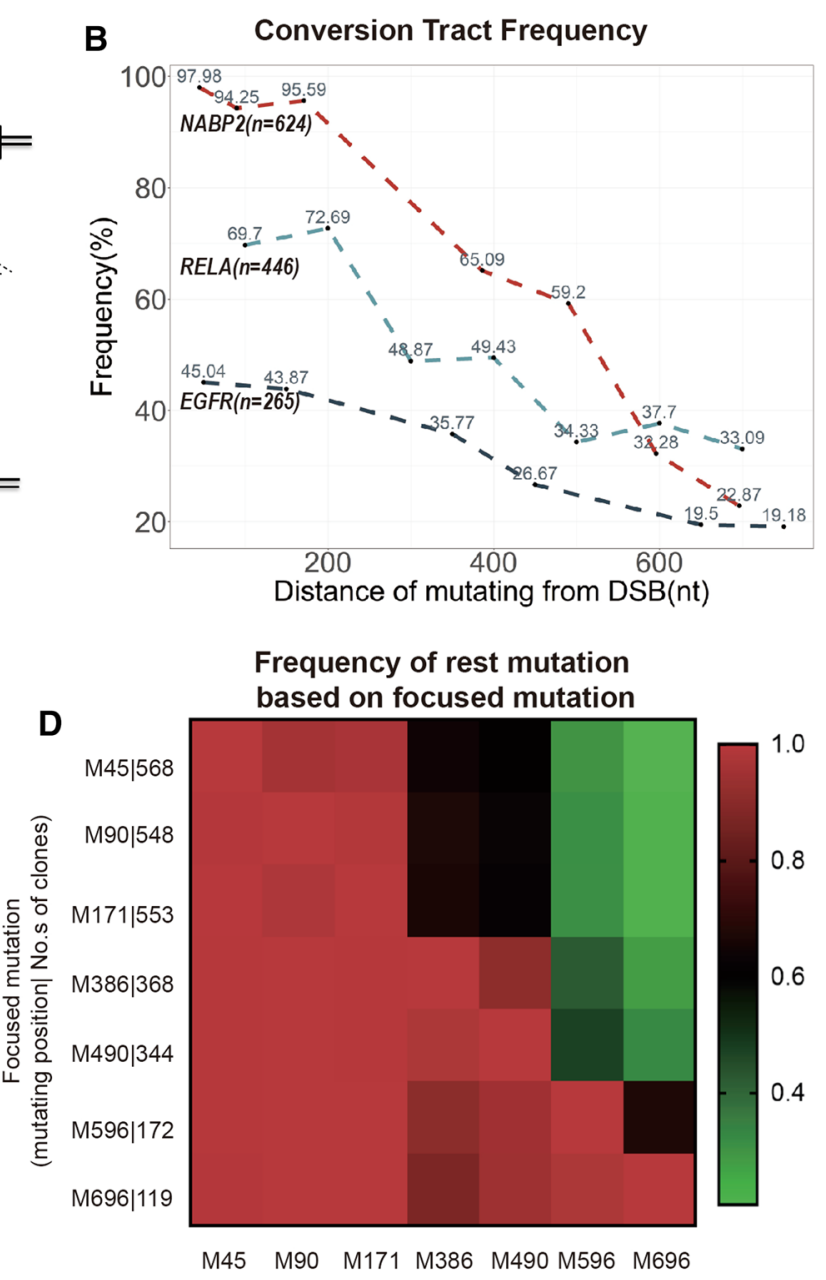

mouse exons (UTR excluded). D Calculation of the selection efficiency of each variation indicated in B from Sanger sequencing of 624 clones that were PCR amplified from the GEIS-edited cell mix. When alteration A (vertical axis, M stands for mutated position, numbers stand for numbers of mutated clones) occurs, the percentage of the remaining alterations (horizontal axis, $\mathrm{M}$ stands for mutated position) is presented on a heatmap

an alteration occurred at the indicated site (vertical axis) (Fig. 3D). According to the map, mutations at a further site largely indicated successful editing of the nearer site, and genome editing showed a high extent of linkage rather than independence, indicating that multiple genome alterations can be introduced in one GEIS reaction.

\section{Discussion}

Here, we have developed a universal and efficient HDRbased gene editing strategy in cell lines. Rather than tagging a fluorescent protein to the target gene ORF for FACS selection, we chose to insert a pCMV-driven DsRed selection marker into introns so that the selection marker did not 
influence target gene expression or splicing when indicating the desired DNA variations for FACS selection. To eliminate the possibility of false-positive cell clone generation by pCMV-driven DsRed-containing donor DNA and random integration of the DNA into the genome via NHEJ, we used ssDNA as the donor [21, 22]. To obtain bulk ssDNA sequences as large as $5000 \mathrm{nt}$ [23], we simply denatured dsDNA at a high temperature in a certain concentration of salt, which is widely applied for all kinds of PCR [24, 25]. Given that an editing target that is too far away from an intron might result in failure to introduce a DNA variation, we explored the editing efficiency based on the conversion tract length, and the results are of great value for other gene editing strategies [26-28]. Considering these results and the analysis of the exon length distribution in humans and mice, we speculate that GEIS can accomplish nearly $97 \%$ of exon editing tasks at relatively high efficiency. In addition, GEIS's ability to introduce multiple DNA variations was also assessed in this study. Such an ability has strong potential for use in the development of novel features based on a directed molecular evolution strategy [29, 30]. Overall, we have introduced a developed gene editing strategy and have described its risks as well as methods to avoid those risks. Our data can also be used in other gene editing applications and can support the use of novel strategies for specific editing tasks.

NHEJ and HDR are two ways to repair DNA after DSBs. The choice is determined by a series of factors, including cell cycle regulation [31] and chromatin context [32]. On the other hand, DSBs could arise during DNA replication, and NHEJ could randomly incorporate dsDNA into the genome in the DNA repair process [33]. Methods to impair NHEJ are useful to increase HDR efficiency [34, 35]. NHEJ inhibitors such as SCR7 increase the HDR product and knocking down SHROOM1 can increase HDR efficiency by 4.7-fold [36]. In this study, both NHEJ and HDR could generate DsRedpositive cells, but only HDR products were desired.

The conversion tract describes the distance of mutation and the DSB in donor DNA [37], which is important in HDR-mediated gene editing. If the conversion tract is too short, the chances of introducing desired DNA alterations are low. The conversion efficiency varies largely in different reports. Previous studies also reported only $20 \%$ when the tract was $200 \mathrm{bp}$ [17]. In plants, the efficacy could remain at $80 \%$ when the tract is $600 \mathrm{bp}$ [38]. In this study, we measured the conversion tract at three different loci, and the efficiency was as high as $65 \%$ for $N A B P 2$ when the tract was $400 \mathrm{bp}$ and $30 \%$ for EGFR. The efficiency could be influenced by the expression of Pifl, Sgsl and Blm [28, 39]. However, these factors cannot explain the difference in different loci in the same cell lines. More factors that influence conversion efficiencies are not yet clear.
This strategy is unlike intronic insertion knock-in HDR strategies, which are restricted to editing of bases near the stop or start codons for tagging markers that must be located at the $\mathrm{N}$ - or $\mathrm{C}$-terminus of a CDS. The independent intronic marker strategy untethers the HDR gene editing strategy, allowing it to be used to solve editing problems in any exon in cells. Although similar intron targeting strategies have been reported, few of them eliminate the burden of in-frame marker tagging [10, 40, 41]. This strategy leaves a DsRed cassette in the genome that may contradict other experiments. To overcome this defect, if needed, we can turn to a scareless gene editing strategy [42] that employs an additional process of HDR to remove the DNA imprint in introns.

\section{Methods}

\section{Plasmid and donor DNA}

DSBs were generated by CRISPR-Cas9 technology with LentiCRISPR-V2 (Addgene, \#98290) carrying the indicated intron-targeting sgRNAs (RELA: GGCUCUGUGCCGUGA GAGAG, NABP2: GGGCAAAGGGGUUUGCAAGG, EGFR: GCCAGCAUUUUCCUGACACC). Important: $s g R$ NAs should avoid targeting the GU-AG at the intron-exon boundaries and the pyrimidines required for RNA splicing! The pDonor-GEIS plasmid was used as the framework for preparing the donor DNA. According to the sequence, two EcoRV sites were located adjacent to the pCMV-driving DsRed cassette for HA cloning. Important: donor DNA should contain no terminators. HAs were PCR amplified from HEK293T genomic DNA and inserted into the plasmid using a Gibson assembly cloning strategy. Mutations in HAs were generated by mutation- or truncation-containing primers using an overlap extension PCR strategy.

Donor DNA was generated from preprepared pDonorGEIS (RELA, NABP2, and EGFR) by PCR using a pair of universal primers: Donor-F: TGTGGTGGAATTCTGCAG AT and Donor-R: GCGGCCGCCACTGTGCTGGAT. PCR was carried out in 28 cycles on an Eppendorf thermocycler with denaturation at $94{ }^{\circ} \mathrm{C}$ for $15 \mathrm{~s}$, annealing at $58{ }^{\circ} \mathrm{C}$ for $15 \mathrm{~s}$, and extension at $72{ }^{\circ} \mathrm{C}$ for 3 min using PrimeS$\operatorname{tar}(\mathrm{TaKaRa}, \mathrm{Japan})$. PCR products were purified using an Ultra-Sep Gel Extraction Kit (Omega).

\section{Fluorescence microscopy}

Donor dsDNA and ssDNA were separately transfected into HEK293T cells. dsDNA was prepared from a purified PCR product that was dissolved in $\mathrm{ddH}_{2} \mathrm{O}$. ssDNA was prepared by denaturation of dsDNA at $95{ }^{\circ} \mathrm{C}$ for $10 \mathrm{~min}$ in $100 \mathrm{mM}$ 
$\mathrm{NaCl}$. Transfected cells were photographed $24 \mathrm{~h}$ after transfection under a fluorescence microscope (NIKON).

\section{Cell culture and FACS}

HEK293T cells were obtained from the American Type Culture Collection. Cells were cultured in DMEM (Dulbecco's modified Eagle's medium) containing $10 \%$ fetal bovine serum at $37{ }^{\circ} \mathrm{C}$ and $5 \% \mathrm{CO}_{2}$. Fifteen micrograms of LentiCRISPR-V2 and $2 \mu \mathrm{g}$ of donor DNA were cotransfected into $5 \times 10^{7}$ cells with Lipofectamine $2000^{\mathrm{TM}}$ (Invitrogen) according to the manufacturer's instructions. Subsequently, transfected cells were treated with $1 \mu \mathrm{g} / \mathrm{mL}$ puromycin for $72 \mathrm{~h}$. Cells were washed with PBS and treated with $0.05 \%$ trypsin. The cell suspension was filtered through a $40 \mu \mathrm{m}$ cell strainer (BD Falcon) before FACS. Flow cytometry analysis and FACS were performed using BD LSR II. Cells isolated by FACS were then cultured for one week and processed by FACS again to enhance the positive rate. Harvested cells were seeded in 96 -well plates at $1 / 2$ cell per well for single-clone growth.

\section{Genomic DNA extraction and analysis}

Genomic DNA was extracted using the TIANamp Genomic DNA Kit (\#DP304-03). PCR of single clones derived from genomic DNA was processed to verify that the clone possessed the desired sequence. PCR was performed for 35 cycles on an Eppendorf thermocycler with denaturation at $94{ }^{\circ} \mathrm{C}$ for $15 \mathrm{~s}$, annealing at $58{ }^{\circ} \mathrm{C}$ for $15 \mathrm{~s}$, and extension at $72{ }^{\circ} \mathrm{C}$ for $30 \mathrm{~s}$ using PrimeStar. Forward primers were located outside the left HA on the genome, and the universal reverse primer was located in the DsRed cassette.

The primers used were as follows:

RELA-gTest-F: GCTCATTGCCAAGGTGGGTA.

NABP2-gTest-F: GGATGGACCGAGTCCCGGCT.

EGFR-gTest-F: ATAAGAAGTCTGCAGAACTT.

Red Uni-R: TTGGACATGACTCCACAT.

\section{Conversion tract length detection}

Multiple DNA variations on the left HA for $N A B P 2$, RELA and EGFR GEIS were introduced to HEK293T cells. FACS-sorted cells were collected for genomic DNA extraction. PCR was performed to amplify the successfully integrated DNA fragment from the donor DNA. PCR was performed for 28 cycles on an Eppendorf thermocycler with denaturation at $94{ }^{\circ} \mathrm{C}$ for $15 \mathrm{~s}$, annealing at $58^{\circ} \mathrm{C}$ for $15 \mathrm{~s}$, and extension at $72{ }^{\circ} \mathrm{C}$ for 1 min using PrimeStar.
PCR products were cloned into the vector $\mathrm{pLV}$-MCS-puroGreen (digested by EcoRI) using Gibson assembly. A total of $E$. coli 624 colonies were sequenced. The mutations identified in the colonies were mapped to the wild-type NABP2 genomic sequence, and the seven candidate DNA alterations were recorded and calculated. The percentage of every variation was calculated as the number of mutated clones divided by 624 (total number of clones) and is illustrated in Fig. 2B.

The primers used were as follows:

EcoRI + NABP2-F: TTCTAGAGCTAGCGAATTGGA TGGACCGAGTCCCGGCT.

EcoRI + Uni-R: CCGATTTAAATTCGAATTTTGGAC ATGACTCCACAT.

\section{Human and mouse exon length distribution}

Human (GRCh38, release 37) and mouse (GRCm38, release M25) genomic annotation files from GENCODE were used to evaluate the distribution of CDSs and exon lengths. In brief, each exon (UTR contained or excluded) was identified, and its length was calculated based on the end and start positions in the genome. The calculated lengths were then grouped and illustrated.

\section{RNA extraction, RT-PCR and qPCR}

Total RNA was extracted using a MolPure Cell/Tissue Total RNA Kit (Yeasen, China). RNA concentration was quantified by Nanodrop ${ }^{\mathrm{C}}$ (Thermo, US). cDNA was processed with DNase treatment and reverse transcription from $500 \mathrm{ng}$ total RNA using the Hifair III 1st Strand cDNA Synthesis Kit (Yeasen). Reverse transcription was performed on a thermocycler at $25{ }^{\circ} \mathrm{C}$ for $5 \mathrm{~min}, 55^{\circ} \mathrm{C}$ for $15 \mathrm{~min}$ and then $85^{\circ} \mathrm{C}$ for $5 \mathrm{~min}$. RT-PCR was processed for 35 cycles on an Eppendorf thermocycler with denaturation at $94{ }^{\circ} \mathrm{C}$ for $15 \mathrm{~s}$, annealing at $58^{\circ} \mathrm{C}$ for $15 \mathrm{~s}$, and extension at $72{ }^{\circ} \mathrm{C}$ for $20 \mathrm{~s}$ using PrimeStar. RT-PCR primers were set on exons adjacent to the processed intron to determine whether any alternative variants were produced. qPCR was performed to check whether there were any significant differences in expression in edited cells. qPCRs were processed using Hieff UNICON Universal Blue qPCR SYBR Green Master Mix (Yeasen) on QuantStudio Dx (ABI), and qPCR primer sequences were derived from PrimerBank. The qPCR was repeated three times.

The RT-PCR and qPCR primers were as follows: 


\section{RT-RELA-F: CTCGGTGGGGATGAGATCTT \\ RT-RELA-R: TTCTTCATGATGCTCTTGAA \\ RT-NABP2-F: GACAAAACAGGCAGCATCAA \\ RT-NABP2-R: GGGTTTGGCTCACTGAAGTT \\ RT-EGFR-F: GTGATGGCCAGCGTGGACAA \\ RT-EGFR-R: GGGATTCCGTCATATGGCTT \\ qGAPDH-F: GGAGCGAGATCCCTCCAAAAT \\ qGAPDH-R: GGCTGTTGTCATACTTCTCATGG}

(product length $=197 \mathrm{bp}$ )

\section{qNABP2-F: TCTGGGACGATGTTGGCAAT qNABP2-R: GGTGCCTGCTGGGTGCTGTA}

$$
\text { (product length }=202 \mathrm{bp} \text { ) }
$$

\section{qRELA-F: CCCAACACTGCCGAGCTCAA qRELA-R: CCTTTTACGTTTCTCCTCAA}

$$
\text { (product length }=348 \mathrm{bp} \text { ) }
$$

\section{qEGFR-F: AGGCACGAGTAACAAGCTCAC qEGFR-R: ATGAGGACATAACCAGCCACC}

$$
\text { (product length }=177 \mathrm{bp} \text { ) }
$$

Supplementary Information The online version contains supplementary material available at https://doi.org/10.1007/s00018-022-04152-1.

Acknowledgements We thank Dr. Kang Wu for technical support in FACS experiments.

Author contributions SWa and YW conceived this idea. SWa performed most of the experiments. YL, SWu and TK provide platform and fundings. $\mathrm{LZ}$ and $\mathrm{KW}$ analysed the data. RZ analysed the sequences.

Funding This project was supported by grants from the National Key Research and Development Program of China [2017YFA0105900 to Song $\mathrm{Wu}$ ], the National Nature Science Foundation of China (NSFC) [81772922 to Y.W., 81802741 to Y. L.], The China Postdoctoral Science Foundation [2020M682949 to Shang Wang]. The Shenzhen Basic Research Project [JCYJ20170412155305340 to Y. L.]. The Special Support Plan for Leading Talents in Scientific and Technological Innovation in Guangdong Province [2017TX04R091 to Song Wu].

Availability of data and material Python script used in this study were online: https://github.com/wukaiyeah/Genomic_CDS_stats.git. FACS data can be downloaded from http://flowrepository.org/id/FR-FCMZ4PY. Sanger sequencing data for conversion tract tests were submitted to GenBank as MZ399804-MZ400408. Sanger sequencing data for conversion tract tests are available in the online version of this manuscript (Supplementary Sanger sequencing data). Sanger sequencing data for conversion tract tests on EGFR and RELA were submitted as attached files. Sanger sequencing data for $N A B P 2$ conversion tract tests were submitted to GenBank as MZ399804-MZ400408.

\section{Declarations}

Conflict of interest The authors declare that they have no competing interests.

Ethics approval Not applicable.

Consent to participate Not applicable.

Consent for publication Not applicable.

Open Access This article is licensed under a Creative Commons Attribution 4.0 International License, which permits use, sharing, adaptation, distribution and reproduction in any medium or format, as long as you give appropriate credit to the original author(s) and the source, provide a link to the Creative Commons licence, and indicate if changes were made. The images or other third party material in this article are included in the article's Creative Commons licence, unless indicated otherwise in a credit line to the material. If material is not included in the article's Creative Commons licence and your intended use is not permitted by statutory regulation or exceeds the permitted use, you will need to obtain permission directly from the copyright holder. To view a copy of this licence, visit http://creativecommons.org/licenses/by/4.0/.

\section{References}

1. Anzalone AV, Koblan LW, Liu DR (2020) Genome editing with CRISPR-Cas nucleases, base editors, transposases and prime editors. Nat Biotechnol 38:824-844. https://doi.org/10.1038/ s41587-020-0561-9

2. Yao X et al (2017) Homology-mediated end joining-based targeted integration using CRISPR/Cas9. Cell Res 27:801-814. https://doi. org/10.1038/cr.2017.76

3. Aird EJ, Lovendahl KN, St Martin A, Harris RS, Gordon WR (2018) Increasing Cas9-mediated homology-directed repair efficiency through covalent tethering of DNA repair template. Commun Biol 1:54. https://doi.org/10.1038/s42003-018-0054-2

4. Chu VT et al (2015) Increasing the efficiency of homologydirected repair for CRISPR-Cas9-induced precise gene editing in mammalian cells. Nat Biotechnol 33:543-548. https://doi.org/10. 1038/nbt.3198

5. Molla KA, Qi Y, Karmakar S, Baig MJ (2020) Base editing landscape extends to perform transversion mutation. Trends Genet 36:899-901. https://doi.org/10.1016/j.tig.2020.09.001

6. Wrighton KH (2019) Cytosine base editors go off-target. Nat Rev Genet 20:254-255. https://doi.org/10.1038/s41576-019-0110-x

7. Zhou $\mathrm{S}$ et al (2019) Programmable base editing of the sheep genome revealed no genome-wide off-target mutations. Front Genet 10:215. https://doi.org/10.3389/fgene.2019.00215

8. Wilkinson ME, Charenton C, Nagai K (2020) RNA splicing by the spliceosome. Annu Rev Biochem 89:359-388. https://doi.org/ 10.1146/annurev-biochem-091719-064225

9. Kocher T et al (2017) Cut and paste: efficient homology-directed repair of a dominant negative KRT14 mutation via CRISPR/Cas9 nickases. Mol Ther 25:2585-2598. https://doi.org/10.1016/j. ymthe.2017.08.015

10. Yoshimi K et al (2021) Combi-CRISPR: combination of NHEJ and HDR provides efficient and precise plasmid-based knock-ins in mice and rats. Hum Genet 140:277-287. https://doi.org/10. 1007/s00439-020-02198-4 
11. Doudna JA, Charpentier E (2014) Genome editing. The new frontier of genome engineering with CRISPR-Cas9. Science 346:1258096. https://doi.org/10.1126/science.1258096

12. Ho TYH et al (2021) A systematic approach to inserting split inteins for Boolean logic gate engineering and basal activity reduction. Nat Commun 12:2200. https://doi.org/10.1038/ s41467-021-22404-9

13. Li PP, Margolis RL (2021) Use of single guided Cas9 nickase to facilitate precise and efficient genome editing in human iPSCs. Sci Rep 11:9865. https://doi.org/10.1038/s41598-021-89312-2

14. Nowak DE et al (2008) RelA Ser276 phosphorylation is required for activation of a subset of NF-kappaB-dependent genes by recruiting cyclin-dependent kinase 9/cyclin T1 complexes. Mol Cell Biol 28:3623-3638. https://doi.org/10.1128/MCB.01152-07

15. Zhang X, Li T, Ou J, Huang J, Liang P (2021) Homologybased repair induced by CRISPR-Cas nucleases in mammalian embryo genome editing. Protein Cell. https://doi.org/10.1007/ s13238-021-00838-7

16. Yoshimi $\mathrm{K}$ et al (2016) ssODN-mediated knock-in with CRISPRCas for large genomic regions in zygotes. Nat Commun 7:10431. https://doi.org/10.1038/ncomms10431

17. Elliott B, Richardson C, Winderbaum J, Nickoloff JA, Jasin M (1998) Gene conversion tracts from double-strand break repair in mammalian cells. Mol Cell Biol 18:93-101. https://doi.org/10. 1128/MCB.18.1.93

18. Pujar $\mathrm{S}$ et al (2018) Consensus coding sequence (CCDS) database: a standardized set of human and mouse protein-coding regions supported by expert curation. Nucleic Acids Res 46:D221-D228. https://doi.org/10.1093/nar/gkx1031

19. Pruitt KD et al (2009) The consensus coding sequence (CCDS) project: Identifying a common protein-coding gene set for the human and mouse genomes. Genome Res 19:1316-1323. https:// doi.org/10.1101/gr.080531.108

20. Harte RA et al (2012) Tracking and coordinating an international curation effort for the CCDS Project. Database (Oxford) 2012:bas008. https://doi.org/10.1093/database/bas008

21. Maurissen TL, Woltjen K (2020) Synergistic gene editing in human iPS cells via cell cycle and DNA repair modulation. Nat Commun 11:2876. https://doi.org/10.1038/s41467-020-16643-5

22. Wang Y et al (2018) Systematic evaluation of CRISPR-Cas systems reveals design principles for genome editing in human cells. Genome Biol 19:62. https://doi.org/10.1186/s13059-018-1445-x

23. Inoue $\mathrm{YU}$ et al (2021) An optimized preparation method for long ssDNA donors to facilitate quick knock-in mouse generation. Cells. https://doi.org/10.3390/cells10051076

24. Ho SN, Hunt HD, Horton RM, Pullen JK, Pease LR (1989) Sitedirected mutagenesis by overlap extension using the polymerase chain reaction. Gene 77:51-59. https://doi.org/10.1016/03781119(89)90358-2

25. Liang P, Pardee AB (1992) Differential display of eukaryotic messenger RNA by means of the polymerase chain reaction. Science 257:967-971. https://doi.org/10.1126/science.1354393

26. Huang TK, Armstrong B, Schindele P, Puchta H (2021) Efficient gene targeting in Nicotiana tabacum using CRISPR/SaCas9 and temperature tolerant LbCas12a. Plant Biotechnol J. https://doi. org/10.1111/pbi.13546

27. Keith $\mathrm{N}$ et al (2016) High mutational rates of large-scale duplication and deletion in Daphnia pulex. Genome Res 26:60-69. https://doi.org/10.1101/gr.191338.115
28. Vernekar DV et al (2021) The Pif1 helicase is actively inhibited during meiotic recombination which restrains gene conversion tract length. Nucleic Acids Res 49:4522-4533. https://doi.org/10. 1093/nar/gkab232

29. Li C et al (2020) Targeted, random mutagenesis of plant genes with dual cytosine and adenine base editors. Nat Biotechnol 38:875-882. https://doi.org/10.1038/s41587-019-0393-7

30. Li C et al (2021) Direct modification of multiple gene homoeologs in Brassica oleracea and Brassica napus using doubled haploid inducer-mediated genome editing system. Plant Biotechnol J. https://doi.org/10.1111/pbi.13632

31. Kass EM, Jasin M (2010) Collaboration and competition between DNA double-strand break repair pathways. FEBS Lett 584:37033708. https://doi.org/10.1016/j.febslet.2010.07.057

32. Ryu $\mathrm{T}$ et al (2015) Heterochromatic breaks move to the nuclear periphery to continue recombinational repair. Nat Cell Biol 17:1401-1411. https://doi.org/10.1038/ncb3258

33. Rothkamm K, Kruger I, Thompson LH, Lobrich M (2003) Pathways of DNA double-strand break repair during the mammalian cell cycle. Mol Cell Biol 23:5706-5715. https://doi.org/10.1128/ MCB.23.16.5706-5715.2003

34. Lin $C$ et al (2016) Increasing the efficiency of CRISPR/Cas9mediated precise genome editing of HSV-1 virus in human cells. Sci Rep 6:34531. https://doi.org/10.1038/srep34531

35. Song J et al (2016) RS-1 enhances CRISPR/Cas9- and TALENmediated knock-in efficiency. Nat Commun 7:10548. https://doi. org/10.1038/ncomms 10548

36. Zhao Z et al (2020) Suppression of SHROOM1 improves in vitro and in vivo gene integration by promoting homology-directed repair. Int J Mol Sci. https://doi.org/10.3390/ijms21165821

37. Ertl HA et al (2017) The role of Blm helicase in homologous recombination, gene conversion tract length, and recombination between diverged sequences in Drosophila melanogaster. Genetics 207:923-933. https://doi.org/10.1534/genetics.117.300285

38. Huang TK, Armstrong B, Schindele P, Puchta H (2021) Efficient gene targeting in Nicotiana tabacum using CRISPR/SaCas9 and temperature tolerant LbCas12a. Plant Biotechnol J 19:1314-1324. https://doi.org/10.1111/pbi.13546

39. Lo YC et al (2006) Sgs1 regulates gene conversion tract lengths and crossovers independently of its helicase activity. Mol Cell Biol 26:4086-4094. https://doi.org/10.1128/MCB.00136-06

40. Serebrenik YV, Sansbury SE, Kumar SS, Henao-Mejia J, Shalem $\mathrm{O}$ (2019) Efficient and flexible tagging of endogenous genes by homology-independent intron targeting. Genome Res 29:13221328. https://doi.org/10.1101/gr.246413.118

41. Suzuki K et al (2019) Precise in vivo genome editing via single homology arm donor mediated intron-targeting gene integration for genetic disease correction. Cell Res 29:804-819. https://doi. org/10.1038/s41422-019-0213-0

42. Ikeda $\mathrm{K}$ et al (2018) Efficient scarless genome editing in human pluripotent stem cells. Nat Methods 15:1045-1047. https://doi. org/10.1038/s41592-018-0212-y

Publisher's Note Springer Nature remains neutral with regard to jurisdictional claims in published maps and institutional affiliations. 